\section{Experiencia de uso de inhibidores del cotransportador sodio-glucosa 2 en diabéticos tipo 2}

\author{
ÁLVARO CONTRERAS ${ }^{1}$, BRUNO GRASSI $^{1}$, DANIELA ALFARO ${ }^{\mathrm{a}}$, \\ FELIPE GONZÁLEZ ${ }^{a}$, VALESKA ORTEGA ${ }^{2}$
}

\section{Use of sodium-glucose cotrasporter 2 inhibitors in type 2 diabetics. Experience in 77 patients}

Background: Sodium-glucose cotransporter 2 inhibitors (SGLT2i) are a new pharmacological alternative for the treatment of diabetes. Aim: To report our experience with the use of this type of drugs in type 2 diabetics treated in an outpatient clinic. Material and Methods: We selected 77 type 2 diabetic patients aged $59 \pm 11$ years ( $45 \mathrm{men}$ ) who started SGLT2i, based on the advice of their treating physician. We registered their demographic characteristics and changes in metabolic parameters, weight, blood pressure, albuminuria and adverse effects, during a follow-up of at least three months. Results: We observed a decrease of glycosylated hemoglobin A1c of $0.8 \pm 1.14 \%(p<0.01)$ and a weight decrease of $2.5 \pm 2.24 \mathrm{~kg}(p<0.01)$. The proportion of patients with a glycosylated hemoglobin A1c of less than $7 \%$ increased from $7.2 \%$ to $30.9 \%(p=0.002)$. In addition, a relative decrease in albuminuria of $39.9 \%$ was observed $(p=0.07)$. The treatment was well tolerated with a rate of adverse effects of $21 \%$, all of them being categorized as mild. Of these, most of them corresponded to genital mycotic infections. Conclusions: The effects observed in this study are comparable and of similar magnitude to randomized studies of SGLT2i reported in the international literature.

(Rev Med Chile 2019; 147: 1093-1098)

Key words: Albuminuria; Diabetes Mellitus, Type 2; Hypoglycemic Agents; Sodium-Glucose Transporter 2 Inhibitors.

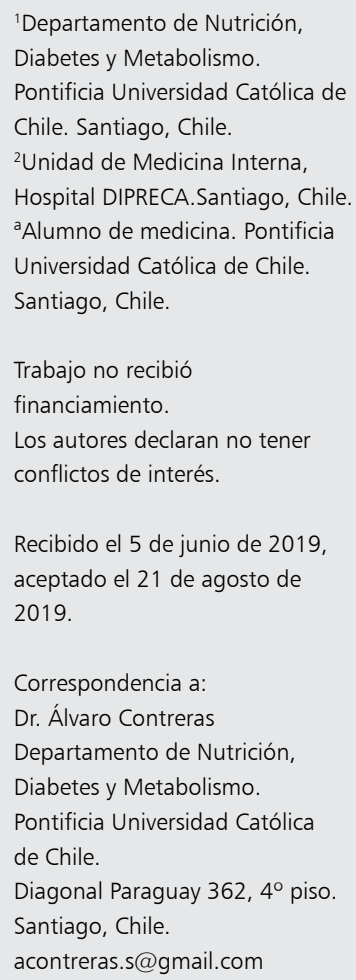

Correspondencia a:

Dr. Álvaro Contreras

Departamento de Nutrición,

Diabetes y Metabolismo.

Pontificia Universidad Católica de Chile.

Diagonal Paraguay $362,4^{\circ}$ piso. Santiago, Chile.

acontreras.s@gmail.com

\section{L} a diabetes mellitus es una enfermedad crónica asociada a una alta frecuencia de complicaciones y morbimortalidad, teniendo alto impacto en la calidad de vida de las personas y en los costos en salud. En la actualidad, se estima que el número de diabéticos en el mundo se acerca a los 500 millones, con una proyección de aumento de casi $50 \%$ en los próximos 30 años $^{1}$. En Chile, según la última encuesta nacional de salud, la prevalencia presunta de diabetes mellitus en la población general sería de $12,3 \%{ }^{2}$.

En el caso de los pacientes con diabetes mellitus tipo 2 (DM2), el manejo de las alteraciones de la glicemia en conjunto con los múltiples factores de riesgo asociados es complejo y altamente demandante en tiempo y recursos, requiriendo, además, un adecuado balance de riesgo/beneficio de las terapias implementadas. La naturaleza progresiva de la enfermedad hace que una alta proporción de los pacientes con DM2 requieran de tratamientos farmacológicos para el control de la hiperglicemia. A pesar de que existen múltiples opciones terapéuticas farmacológicas, debe realizarse una correcta selección de la terapia de acuerdo a las caracterís- 
ticas individuales de cada paciente, indicaciones y contraindicaciones, expectativas y pronóstico vital, costos y disponibilidad, entre otros ${ }^{3}$.

Las opciones farmacológicas disponibles para el tratamiento de la DM2 se han incrementado extraordinariamente durante los últimos 20 años. El abanico de fármacos actuales permite abarcar distintos mecanismos de acción y diferentes perfiles de efectividad, siendo la mayoría de ellos dependientes de la acción de la insulina para su efecto $^{3-5}$. Sin embargo, este mayor número de alternativas terapéuticas no se ha traducido necesariamente en una mejoría en el nivel de control metabólico de los pacientes diabéticos, muchos de los cuales no logran su objetivo de hemoglobina glicosilada, acarreando un riesgo significativo de complicaciones micro y macrovasculares ${ }^{6,7}$.

Los inhibidores del cotransportador sodio-glucosa 2 (SGLT2) son una nueva clase farmacológica para el tratamiento de la diabetes mellitus tipo 2, cuyo mecanismo de acción es independiente de la secreción y acción de la insulina. La inhibición del SGLT2 a nivel del túbulo proximal del nefrón produce una disminución en la reabsorción tubular de la glucosa filtrada a nivel glomerular, disminuyendo significativamente el umbral de glucosuria en relación a la glicemia plasmática ${ }^{8,9}$. El aumento en la excreción urinaria de glucosa se traduce en una disminución efectiva de la glicemia plasmática, siendo su principal mecanismo de acción como fármaco antidiabético ${ }^{10}$.

En el presente trabajo presentamos la experiencia clínica en nuestro centro con el uso de inhibidores SGLT2 en pacientes con DM2, evaluando como objetivos principales el impacto en control glicémico, beneficios extraglicémicos y efectos adversos.

\section{Materiales y Métodos}

Estudio observacional realizado en pacientes ambulatorios atendidos dentro de la red de salud de la Universidad Católica de Chile. El diseño fue aprobado por el Comité de Ética de la Escuela de Medicina de la Pontificia Universidad Católica de Chile.

\section{Pacientes}

Se seleccionaron pacientes adultos (mayores de 18 años), con diagnóstico de diabetes mellitus, que iniciaron tratamiento con algún inhibidor de SGLT2 por indicación de su médico tratante. No se restringió por edad ni hemoglobina glicosilada, así como tampoco por otras terapias antidiabéticas usadas previo al inicio del fármaco.

Se excluyeron los pacientes con diagnóstico previo o sospecha de diabetes mellitus tipo $1, \mathrm{y}$ aquellos que iniciaron en forma simultánea con el fármaco estudiado otros fármacos con efecto hipoglicemiante, incluyendo metformina, sulfonilureas, inhibidores DPP-4, tiazolidinedionas, análogos GLP-1, y cualquier tipo de terapia con insulina, ya sea en forma transitoria o permanente.

Se realizó registro en forma anónima de variables demográficas del grupo estudiado, como edad y sexo, parámetros antropométricos (peso, talla e índice de masa corporal), presión arterial, indicadores de control glicémico previo (hemoglobina glicosilada), presencia de complicaciones microvasculares conocidas (retinopatía, nefropatía y neuropatía diabética), enfermedad cardiovascular establecida (cardiopatía coronaria, accidente cerebrovascular y enfermedad arterial periférica), hipertensión arterial, índice de microalbuminuria/ creatininuria en muestra de orina aislada. Además, se realizó un registro detallado de todos los fármacos antidiabéticos usados por el paciente durante el período de observación.

\section{Seguimiento}

El seguimiento se realizó según criterio e indicación del médico tratante, incluyéndose en el análisis a pacientes que tuvieran tres o más meses de seguimiento, con datos de al menos una variable de estudio, independiente del uso o suspensión del fármaco.

\section{Eventos analizados}

El evento primario analizado fue el cambio de hemoglobina glicosilada, luego de 3 meses o más de tratamiento con inhibidor de SGLT2, respecto del valor previo al tratamiento, así como la proporción de pacientes que alcanzaron hemoglobina glicosilada menor a 7\%. Además, se analizó en forma secundaria el cambio de peso, presión arterial sistólica, diastólica y media, e índice de microalbuminuria/creatininuria en muestra aislada.

\section{Eventos adversos}

Se registraron todos los efectos adversos atribuibles al uso del fármaco, quedando la adjudi- 
cación del evento a criterio del médico tratante, así como también las modificaciones de dosis y suspensiones de tratamiento. En caso de suspensión, se registró el motivo detallado clasificándolo como relacionado o no a efecto adverso, según registro clínico del médico tratante.

\section{Análisis estadístico}

Para efectos descriptivos de la población estudiada se calcularon los promedios y desviación estándar de los distintos parámetros evaluados al inicio del tratamiento y luego de 3 meses. Se realizaron comparaciones pre y postratamiento mediante test de $t$ de Student para datos pareados, mientras que las proporciones de pacientes que cumplieron A1c menor a 7\% se compararon con test exacto de Fisher. Se consideraron diferencias estadísticamente significativas las con un valor $\mathrm{p}<0,05$.

\section{Resultados}

Se incluyeron en el análisis final a 77 pacientes que iniciaron algún inhibidor SGLT2 durante el período estudiado y se mantuvieron en seguimiento por al menos 3 meses. Las características de los pacientes al momento del inicio del tratamiento se detallan en la Tabla 1. Cabe destacar que el promedio de tiempo de evolución de diabetes fue mayor de 10 años, así como una alta proporción de los pacientes con presencia de complicaciones microvasculares (61\%) y uso de insulina $(68,8 \%)$.

La distribución de los fármacos inhibidores SGLT2 usados en esta serie fue: dapagliflozina (Forxiga ${ }^{\circledR}$ ) 79,2\%, empagliflozina (Jardiance ${ }^{\circledR}$ ) $18,2 \%$ y canagliflozina (Invokana ${ }^{\circledR}$ ) $1,3 \%$.

En la Tabla 2 se resumen los principales resultados del estudio. Luego del uso de inhibidor SGLT2 se observó una disminución promedio de hemoglobina glicosilada de $0,8 \pm 1,14 \%$ $(\mathrm{p}<0,001)$, asociado a una disminución promedio significativa de peso de $2,5 \pm 2,24 \mathrm{~kg}(\mathrm{p}<0,001)$. La proporción de pacientes con hemoglobina glicosilada menor a $7 \%$ previo al tratamiento fue de $7,2 \%$, mientras que postratamiento alcanzó a $30,9 \%(p=0,002)$. No se demostraron diferencias significativas en parámetros de presión arterial sistólica, presión arterial diastólica, ni función renal. Luego del tratamiento, la albuminuria presentó una disminución relativa de 39,9\%, siendo esta diferencia estadísticamente significativa $(p=0,007)$. En el subgrupo de pacientes con albuminuria mayor $30 \mathrm{mg} / \mathrm{g}$ creatinina (16 pacientes), la albuminuria promedio previa al inicio de tratamiento fue de $126,6 \mathrm{mg} / \mathrm{g}$ creatinina, disminuyendo luego del uso de inhibidores de SGLT2 a 73,2 mg/g creatinina, traduciendo una reducción relativa de $42,1 \%(\mathrm{p}=0,007)$.

En relación a efectos adversos, se observaron en $21 \%$ de los pacientes, siendo todos categorizados como de carácter leve. La reacción adversa más frecuentemente reportada fue la infección micótica genital, que ocurrió en $15,1 \%$ del total de los pacientes. No se observaron efectos adversos graves atribuibles al uso del fármaco, como cetoacidosis diabética o amputaciones. Seis pacientes suspendieron el tratamiento con inhibidores de SGLT2, de los cuales, 5 fueron por eventos recurrentes de micosis genital. El detalle de los efectos adversos registrados se adjunta en la Tabla 3.

Tabla 1. Características de los pacientes al inicio del tratamiento

\begin{tabular}{|c|c|}
\hline \multicolumn{2}{|l|}{ Variable } \\
\hline Edad - años* & $59,2 \pm 10,8$ \\
\hline Duración de diabetes - años* & $12,3 \pm 6,9$ \\
\hline Peso - kg* & $83,6 \pm 14,7$ \\
\hline $\mathrm{IMC}-\mathrm{kg} / \mathrm{m}^{2 *}$ & $30,7 \pm 4,6$ \\
\hline $\mathrm{Hb} \mathrm{A} 1 \mathrm{c}-\%$ * & $8,6 \pm 1,38$ \\
\hline Hombres - n (\%) & $45(58,4)$ \\
\hline HTA - n (\%) & $53(68,8)$ \\
\hline Complicación microvascular - n (\%) & $47(61)$ \\
\hline - Nefropatía & $22(28,5)$ \\
\hline - Retinopatía & $23(29,8)$ \\
\hline - Neuropatía & $19(24,6)$ \\
\hline Enfermedad Cardiovascular - n (\%) & $22(28,6)$ \\
\hline Otros hipoglicemiantes - n(\%) & $73(94,8)$ \\
\hline - Metformina & $68(88,3)$ \\
\hline - Inhibidor DPP-4 & $27(35)$ \\
\hline - Otro & $13(16,8)$ \\
\hline Insulinoterapia - n (\%) & $53(68,8)$ \\
\hline
\end{tabular}

* Valores expresados en promedio \pm desviación estándar. $\mathrm{HTA}=$ hipertensión arterial; IMC = índice de masa corporal. 
Tabla 2. Resultados principales

\begin{tabular}{|lccc|}
\hline Parámetro & Pretratamiento & Postratamiento & p \\
\hline HbA1c - \% & 8,44 & 7,64 & $<0,001$ \\
\hline HbA1c $<7 \%$ - $n(\%)$ & $4(7,2)$ & $17(30,9)$ & 0,002 \\
\hline Presión arterial sistólica - mmHg & 131,4 & 128,3 & 0,16 \\
\hline Presión arterial diastólica - mmHg & 77,8 & 78 & 0,90 \\
\hline Peso - kg & 82,7 & 80,2 & $<0,001$ \\
Albuminuria - mg/g creatinina & 54,1 & 32,5 & 0,007 \\
\hline Albuminuria $>30$ mg/g creatinina & 126,6 & 73,2 & 0,007 \\
\hline
\end{tabular}

Tabla 3. Efectos adversos

\begin{tabular}{|lc|}
\hline Efecto adverso & $\mathbf{n}(\mathbf{\%})$ \\
\hline Todos & $14(21,2)$ \\
\hline Micosis genital & $10(15,1)$ \\
\hline Infección urinaria baja & $2(3,0)$ \\
\hline Urgencia miccional & $1(1,51)$ \\
\hline Síntomas gastrointestinales & $1(1,51)$ \\
\hline
\end{tabular}

\section{Discusión}

El presente estudio tuvo como objetivo mostrar la experiencia con el uso de inhibidores SGLT2 en una población de pacientes con DM2, en el ámbito nacional. Al ser nuestro centro uno de referencia de atención de especialidad, observamos que nuestro grupo de pacientes analizados eran de alta complejidad desde el punto de vista de su diabetes: la mayoría con más de 10 años de evolución de enfermedad, con alta prevalencia de complicaciones micro y macrovasculares, alto número de comorbilidades y, la mayoría, usuarios de insulina. En este contexto, nuestros pacientes parecen ser de mayor nivel de complejidad que la gran mayoría de los estudios primarios internacionales de inhibidores SGLT2 ${ }^{11,12}$.

En un gran número de estudios de distribución aleatoria comparado contra placebo, los inhibidores SGLT2 han demostrado efectividad en mejoría de control metabólico en pacientes DM2, logrando reducciones de $\mathrm{HbAlc}$ de entre 0,5 y $1,0 \%{ }^{12,13}$. En un metaanálisis de 17 estudios la reducción media de HbA1c con el uso de inhibidores de SGLT2 fue de $0,7 \%{ }^{13}$. Nuestros resultados, en contexto de vida real son concordantes con la evidencia publicada, mostrando una reducción promedio de $\mathrm{HbAlc}$ de $0,8 \%$, así como un aumento significativo de la proporción de pacientes que lograron $\mathrm{HbAlc}$ menor a 7\%, con un seguimiento de al menos 3 meses.

Por otra parte, diversos estudios han mostrado una serie de efectos extraglicémicos beneficiosos con el uso de inhibidores SGLT2. Un metaanálisis mostró una reducción de peso promedio de $2,2 \mathrm{~kg}$ en comparación al uso de inhibidores de DPP4 $4^{14}$, mientras que una revisión sistemática evidenció bajas de peso medias respecto a placebo de $1,8 \mathrm{~kg}$ para dapagliflozina, 2,3 $\mathrm{kg}$ para canagliflozina $\mathrm{y}$ 2,0 kg para empagliflozina ${ }^{15}$. En nuestro estudio, en ausencia de un grupo control, se observó un efecto similar a estos datos ya publicados, con una baja de peso promedio de $2,5 \mathrm{~kg}$. Respecto a efectos en la presión arterial, está descrito en la literatura bajas de entre 3 y $5 \mathrm{mmHg}$ para presión sistólica, y de alrededor de $2 \mathrm{mmHg}$ para presión diastólica ${ }^{16}$. Sin embargo, en nuestra serie no logramos mostrar cambios significativos en las cifras, tanto de presión arterial sistólica como diastólica con el uso de inhibidores SGLT2. Una probable explicación para esta discrepancia es el carácter observacional de nuestro estudio, por lo cual el médico tratante podía ajustar el tratamiento antihipertensivo del paciente según necesidad, tanto antes como después del inicio del inhibidor SGLT2.

Se logró demostrar, además, un efecto significativo en reducción de las cifras de microalbuminuria, que, si bien pueden ser atribuibles a cointervenciones o mejoría en el control metabólico, también son concordantes con lo mostrado en estudios clínicos con distribución aleatoria con inhibidores de SGLT2 $2^{17-20}$. 
En cuanto a efectos adversos, la gran mayoría están relacionados a un incremento en el riesgo de infecciones micóticas genitales. En este contexto, nuestra observación es concordante con otros estudios, que muestran un incremento de alrededor de $300 \%$ en la incidencia de este tipo de infecciones en los pacientes usuarios de inhibidores SGLT2 ${ }^{21}$. No observamos eventos adversos infecciosos serios, así como tampoco episodios de cetoacidosis diabética, los cuales han sido descritos en la literatura en relación al uso de esta clase farmacológica ${ }^{22}$. Sin embargo, el número de pacientes incluidos en este análisis y el período de observación limitado no permiten evaluar la ocurrencia de eventos de baja frecuencia.

Identificamos diversas limitaciones de nuestro estudio, siendo la más relevante su metodología observacional y ausencia de grupo control, que impide aislar el efecto de la intervención farmacológica en estudio de otras posibles intervenciones no reportadas. Por otra parte, el tiempo de seguimiento es variable según la asistencia a controles médicos de los pacientes, por lo que no es posible afirmar que los efectos observados en nuestra cohorte son duraderos en el tiempo. Las principales fortalezas de nuestro estudio son su carácter local, siendo la primera experiencia reportada en el ámbito nacional con este tipo de fármacos, el incluir pacientes diabéticos de alto grado de complejidad que son más susceptibles a presentar efectos adversos o menor beneficio de intervenciones con fármacos orales, y el estar restringido a un centro de referencia con labor docente, en el cual existe cierto grado de uniformidad en los criterios y directrices de manejo generales de los pacientes diabéticos tipo 2.

Concluimos del presente estudio que los efectos metabólicos y extraglicémicos de los inhibidores de SGLT2 en un grupo de pacientes chilenos con DM2 son concordantes con los observados en los estudios internacionales y, por lo tanto, es muy probable que la nueva evidencia obtenida en relación a esta clase farmacológica sea extrapolable a futuro a nuestra población.

\section{Referencias}

1. Cho NH, Shaw JE, Karuranga S, Huang Y, da Rocha Fernandes JD, Ohlrogge AW et al. IDF Diabetes Atlas: Global estimates of diabetes prevalence for 2017 and projections for 2045. Diabetes Res Clin Pract 2018; 138: 271-81.

2. Documento presentación primeros resultados Tercera Encuesta Nacional de Salud (ENS) 2016-2017. Disponible en: http://epi.minsal.cl/wpcontent/uploads/ 2017/12/2017.21.07_pdf.primeros.resultados.pdf.

3. Inzucchi SE, Bergenstal RM, Buse JB, Diamant M, Ferrannini E, Nauck M, et al. Management of hyperglycemia in type 2 diabetes, 2015: a patient-centered approach: update to a position statement of the American Diabetes Association and the European Association for the Study of Diabetes. Diabetes Care 2015; 38: 140-9.

4. Rodbard HW, Jellinger PS, Davidson JA, Einhorn D, Garber AJ, Grunberger G, et al. Statement by an American Association of Clinical Endocrinologists/American College of Endocrinology consensus panel on type 2 diabetes mellitus: an algorithm for glycemic control. Endocr Pract 2009; 15 (6): 540-59.

5. American Diabetes Association. Pharmacologic Approaches to Glycemic Treatment: Standards of Medical Care in Diabetes-2018. Diabetes Care 2018; 41 (Suppl 1): S73-S85.

6. CDC. National Diabetes Statistics Report, 2017. Disponible en: https://www.cdc.gov/diabetes/pdfs/data/ statistics/national-diabetes-statistics-report.pdf.

7. Nwaneri C, Cooper H, Bowen-Jones D. Mortality in type 2 diabetes mellitus: magnitude of the evidence from a systematic review and meta-analysis. Br J Diabetes Vasc Dis 2013; 13: 192-207.

8. DeFronzo RA, Davidson JA, del Prato S. The role of the kidneys in glucose homeostasis: a new path towards normalizing glycaemia. Diabetes Obes Metab 2012; 14 (1): 5-14.

9. Nair S, Wilding JP. Sodium glucose cotransporter 2 inhibitors as a new treatment for diabetes mellitus. J Clin Endocrinol Metab 2010; 95 (1): 34-42.

10. Ferrannini E, Muscelli E, Frascerra S, Baldi S, Mari A, Heise $\mathrm{T}$, et al. Metabolic response to sodium-glucose cotransporter 2 inhibition in type 2 diabetic patients. J Clin Invest 2014; 124 (2): 499-508.

11. Liu XY, Zhang N, Chen R, Zhao JG, Yu P. Efficacy and safety of sodium-glucose cotransporter 2 inhibitors in type 2 diabetes: a meta-analysis of randomized controlled trials for 1 to 2 years. J Diabetes Complications. 2015; 29 (8): 1295-303.

12. Shyangdan DS, Uthman OA, Waugh N. SGLT-2 receptor inhibitors for treating patients with type 2 diabetes mellitus: a systematic review and network meta-analysis. BMJ Open 2016; 6 (2): e009417.

13. Berhan A, Barker A. Sodium glucose co-transport 2 inhibitors in the treatment of type 2 diabetes mellitus: 
a meta-analysis of randomized double-blind controlled trials. BMC Endocr Disord 2013; 13: 58.

14. Zaccardi F, Webb DR, Htike ZZ, Youssef D, Khunti $\mathrm{K}$, Davies MJ. Efficacy and safety of sodium-glucose co-transporter-2 inhibitors in type 2 diabetes mellitus: systematic review and network meta-analysis. Diabetes Obes Metab 2016; 18 (8): 783-94.

15. Cai X, Yang W, Gao X, Chen Y, Zhou L, Zhang S, et al. The Association Between the Dosage of SGLT2 Inhibitor and Weight Reduction in Type 2 Diabetes Patients: A Meta-Analysis. Obesity 2018; 26 (1): 70-80.

16. Baker WL, Buckley LF, Kelly MS, Bucheit JD, Parod ED, Brown R, et al. Effects of Sodium-Glucose Cotransporter 2 Inhibitors on 24-Hour Ambulatory Blood Pressure: A Systematic Review and Meta-Analysis. J Am Heart Assoc. 2017 May 18;6(5).

17. Cherney D, Lund SS, Perkins BA, Groop PH, Cooper ME, Kaspers S, et al. The effect of sodium glucose cotransporter 2 inhibition with empagliflozin on microalbuminuria and macroalbuminuria in patients with type 2 diabetes. Diabetologia 2016; 59 (9): 1860-70.
18. Fioretto P, Stefansson BV, Johnsson E, Cain VA, Sjöström CD. Dapagliflozin reduces albuminuria over 2 years in patients with type 2 diabetes mellitus and renal impairment. Diabetologia 2016; 59 (9): 2036-9.

19. Wanner C, Inzucchi SE, Lachin JM, Fitchett D, von Eynatten M, Mattheus M, et al. Empagliflozin and Progression of Kidney Disease in Type 2 Diabetes. N Engl J Med 2016; 375 (4): 323-34.

20. Neal B, Perkovic V, Mahaffey KW, de Zeeuw D, Fulcher $\mathrm{G}$, Erondu $\mathrm{N}$ et al. Canagliflozin and Cardiovascular and Renal Events in Type 2 Diabetes. N Engl J Med 2017; 377 (7): 644-57.

21. Liu J, Li L, Li S, Jia P, Deng K, Chen W et al. Effects of SGLT2 inhibitors on UTIs and genital infections in type 2 diabetes mellitus: a systematic review and meta-analysis. Sci Rep 2017; 7 (1): 2824.

22. Peters AL, Buschur EO, Buse JB, Cohan P, Diner JC, Hirsch IB. Euglycemic Diabetic Ketoacidosis: A Potential Complication of Treatment With Sodium-Glucose Cotransporter 2 Inhibition. Diabetes Care 2015; 38 (9): 1687-93. 